\title{
The beautiful structures of BAFF, APRIL and their receptors
}

Pascal Schneider

Department of Biochemistry, University of Lausanne, CH-1066 Epalinges, Switzerland.

Running title: Structure of BAFF and APRIL

Word count: 6118

Keywords: Structure, B cells, oligomerization, antibody, survival, receptor, ligand, TNF.

Abbreviations:

APRIL: A PRoliferation-Inducing Ligand; BAFF: B cell Activating Factor of the TNF

Family; BCMA: B Cell Maturation Antigen; TACI: Transmembrane Activator and CAML-Interactor.

Corresponding author: Pascal Schneider

Biochemistry Department, University of Lausanne

Boveresses 155

CH-1066 EPALINGES, Switzerland

Phone: $\quad$ +41216925709 Fax:+41 216925705

e-mail pascal.schneider@unil.ch 


\begin{abstract}
The TNF family ligands BAFF/BLyS and APRIL play important roles in the homoestasis and function of $\mathrm{B}$ cells. BAFF binds to the receptors BAFF-R/BR3, BCMA and TACI, whereas APRIL interacts with BCMA, TACI and sulfated side chains of proteoglycans. BAFF and APRIL are initially synthetized as membrane-bound proteins that can be released into soluble forms by proteolytic processing. Both cytokines display the characteristic homotrimeric structure of the TNF family, but BAFF is further able to oligomerize under certain conditions into a virus-like particle containing twenty trimers. All three receptors for BAFF are smaller than canonical TNF receptors and form compact but extensive interactions with the ligands. Similarities and differences in these interactions account for the observed binding specificities to BAFF and APRIL. In contrast to TACI and BCMA, proteoglycans interact with APRIL at distinct sites and may serve to concentrate APRIL at defined anatomical locations. Multimeric forms of BAFF and APRIL are required for signaling through TACI, whereas BAFF-R responds to all forms of BAFF, suggesting that the many different types of BAFF and APRIL may be functionally relevant to control distinct aspects of their biology.
\end{abstract}




\section{Introduction}

The cytokines B cell Activating Factor of the TNF Family (BAFF, also known as BLyS or TALL-1) and A PRoliferation Inducing Ligand (APRIL) regulate several aspects of B cell function and homeostasis. BAFF-deficient mice have little mature B cells in the periphery and impaired humoral responses, whereas BAFF transgenic mice present with B cell hyperplasia, hyperglobulinemia and autoimmunity (1-3). The phenotype of APRIL-deficient mice is less severe, with deficient $\operatorname{IgA}$ responses to mucosal immunization, whereas APRIL transgenic mice have elevated $\operatorname{Ig} \mathrm{A}$ levels and can develop chronic lymphoblastic leukemia-like tumors derived from the B1 B cell population $(4,5)$. BAFF binds to three receptors, BAFF-R, BCMA and TACI, whereas APRIL binds BCMA and TACI only (reviewed in $(6,7)$ ) (Figure 1). The phenotype of BAFF-Rdeficient mice is similar to that of BAFF-null mice, with marked decrease of mature B cells $(8,9)$. BCMA-null mice are essentially normal, but with impaired survival of bone marrow plasma cells (10). Finally, TACI is essential for humoral responses to Tindependent type-2 antigens, but also display B cell hyperplasia, indicating that TACI exerts a negative role on the peripheral B cell population (11-13). It is unclear whether the negative role of TACI on B cells is direct or indirect.

In human, mutations in TACI are found in about $8 \%$ of patients with common variable immunodeficiency, a condition characterized by low antibody levels and susceptibility to infections (14-17). BAFF is also an essential B cells survival factor in birds, although BAFF-producing cells are different in birds and mammals (18). Curiously, APRIL appears to be missing in birds. In contrast, fishes do not only express BAFF and APRIL, but also a third related ligand named BALM (for BAFF-APRIL-like molecule), which is absent in mammals (19).

In the present chapter, structural aspects of BAFF, APRIL and their receptors will be reviewed.

\section{Multiple forms of BAFF and APRIL}

BAFF and APRIL are remarkable by the diversity of their mature forms that result both from splicing and post-translational events. The numerous splice variants have been reviewed previously (7). Briefly, delta-BAFF lacks exon 3 and acts as a dominant 
negative inhibitor of the active full-length form (20). Similar exon skipping events occur in human APRIL, but their functional impacts have not been characterized. Moreover, an intergenic splicing between tweak and april genes generate TWE-PRIL, a hybrid ligand containing the full receptor-binding domain of APRIL (21) (Figure 1). Finally, the use of an alternative splice acceptor site generates two isoforms of mouse APRIL differing by just one amino acid (Ala120). These two isoforms only display subtle differences in receptor binding, and the shorter isoform does not exist in human (22).

BAFF and APRIL are both synthesized as membrane-bound proteins that can be processed to soluble forms by proteolytic processing at a furin consensus site $(23,24)$ (Figure 2). APRIL is efficiently processed within the cell, and whether APRIL can be expressed as a membrane-bound form remains to be demonstrated (25). However, TWEPRIL was suggested to exist in a membrane-bound form (21).

Soluble BAFF can assemble as virus-like particles (26), and soluble APRIL can bind to proteoglycans $(27,28)$ (Figure 1). Formation of BAFF-APRIL heteromers has also been reported in sera of patients with rheumatoid arthritis (29).

\section{Structure of BAFF and APRIL}

\subsection{Structural comparison with other of TNF family members}

TNF and related ligands assemble as homotrimers (30), and BAFF and APRIL are no exception to the rule (31-33) (Figure 3). Each APRIL and BAFF protomers are composed of two $\beta$-sheets containing strands A'AHCF and B'BGDE. Strands A, C, E, F and $\mathrm{H}$ are the most conserved and form much of the trimer interface. This interface is rich in hydrophobic interactions that appear to be the main forces driving trimer formation. The B'BGDE sheet is more solvent exposed and most of its strands are less conserved in sequence among different TNF family ligands (30). BAFF and APRIL have an internal disulfide bridge linking strands $\mathrm{E}$ and $\mathrm{F}$ (Figure 2). This later feature is shared with the TNF family ligands BALM, EDA and Tweak.

\subsection{BAFF forms virus-like particles}

In some studies, BAFF crystallized as a virus-like particle containing 20 trimers $(26,34$, 35). The BAFF 60-mer contains 3 -fold symmetry axes corresponding to those of each 
BAFF 3-mer, but also 5-fold symmetry axes (Figure 4; colored ring of 5 BAFF 3-mers). In this structure, BAFF trimers interact with each other through extended sets of interactions involving the unusually long DE-loop, also known as the "flap". There are numerous interactions in this region involving salt bridges and hydrophobic interactions (Figure 4, lower panels). In one published structure, two citrate and three magnesium ions interact with the portion of the flap facing the inside of the 60-mer structure (32). The flap and most of the key residues involved in trimer-trimer interactions are conserved in BAFF from vertebrates (Figure 2). These features are not conserved in APRIL, BALM and other TNF family members (Figure 2). The high number of charged interactions required for BAFF 60 -mer assembly explains the acid-sensitivity of this structure $(26,36)$ and the different outcome of crystallization performed at acidic or neutral $\mathrm{pH}(26,32,33$, 37). Mutation of His 218 to Ala entirely prevented 60-mer formation in human BAFF (36).

\subsection{APRIL binds to proteoglycans}

Proteoglycans are heavily glycosylated O-linked glycoproteins that are either soluble or membrane-bound (38). They have extended, negatively charged oligosaccharide side chains collectively designated glycosaminoglycans. Negative charges originate from anionic monosaccharides (such as glucuronic or iduronic acids) and from more or less extensive sulfation events. Heparin is a protein-free glycosaminoglycan of the heparan sulfate type.

Human and mouse APRIL contain a short basic amino acid sequence preceding $\beta$-strand A (Figure 2). This basic sequence, which is absent in BAFF, is required but not sufficient to bind heparin and proteoglycans $(27,28)$. Additional basic residues scattered on the same surface of APRIL are also required for binding to heparin (27). It is therefore likely that negatively charged glycosaminoglycans bind to a basic surface of APRIL, as illustrated in the model shown in Figure 5. It is noteworthy that the proteoglycan-binding site of APRIL is clearly distinct from the binding sites of TACI and BCMA (Figure 5).

\section{Receptor binding}

\subsection{Structure of BCMA, TACI and BAFF-R}


TNF receptor family members are generally type I transmembrane proteins with cysteinrich domains (CRDs) in their extracellular domains. These CRDs can be further divided into small structural units called modules (30). For example, TNF-R1 contains 4 CRDs corresponding to a total of eight modules. BCMA, TACI and BAFF-R differ significantly form canonical TNF receptors. First, they are type III trans-membrane proteins lacking a signal peptide. Second, TACI contains two CRDs, BCMA has only one and BAFF-R has just no canonical CRD. Third, they contain a module not present in other TNF receptors and thus are not easy to recognize as such based on the primary sequence only. BAFF-R, BCMA and TACI share a structurally conserved $\beta$-hairpin (corresponding to module A1 of the third CRD of TNF-R1) immediately followed by a short, one turn helix (Figure 6). This determines much of the ligand binding properties. After these conserved structural features, receptors diverge: TACI and BCMA have an additional helix, but that adopt different orientations (39) (Figure 7). The second module of BAFF-R is truncated and the one remaining cystein residue at the end of the one turn helix pairs with another cystein residue in the $\beta$-hairpin (Figure 6).

TACI is remarkable in that it contains 2 CRDs that probably arose from exon duplication (39). CRD2 binds BAFF and APRIL with high affinity, whereas CRD1 also binds, but with low affinity. In human, a short form of TACI lacking CRD1 is produced by alternative splicing (39) (Figure 1). It is noteworthy that TACI, like APRIL, binds to proteoglycans (40) (Figure 1). The site of this interaction has not been mapped.

\subsection{Comparison of ligand-receptor binding in the TNF family}

The TNF receptor family members TNF-R1, OX40 and DR5 are elongated proteins that contact their ligands at the interface between two protomers (41-44) (Figure 8). The interaction extends over the entire length of the ligand and involves residues of both ligand protomers (Figure 8). In contrast, the contact site of BAFF-R on BAFF is localized and involves mainly a single BAFF protomer (Figure 8). The same is true for BCMA and TACI binding to BAFF and APRIL (Figure 8). The surface buried by interaction with an extended receptor (like TNF-R1) or a compact receptor (like BAFF-R) is approximately the same, so that both types of interaction can be of high affinity. As mentioned above, the binding of BCMA or TACI to APRIL does not overlap with the proteoglycan-binding 
site of APRIL (Figure 5). In BAFF 60-mer, the receptor binding sites remain accessible on the outer surface of the virus-like cluster (Figure 4). In this structure however, the receptor-binding pocket of BAFF is delimitated by the flap region of the neighboring BAFF trimer, raising the question of how the long form of TACI does bind this structure. If the CRD1-CRD2 junction of TACI is flexible enough, the high affinity CRD2 will probably bind BAFF 60-mer. If this junction is rigid, only the low affinity CRD1 of TACI may have access to the binding site.

\subsection{Structural determinants of binding specificity}

The $\beta$-hairpins of BCMA, BAFF-R and TACI contain conserved aspartate and leucine residues known as the DxL motif (Figure 6). A synthetic, constrained peptide containing just 6 residues of BAFF-R hairpin, including the DxL motif, is sufficient for BAFF binding (45). In an experimental procedure called shotgun alanine scanning, residues of the receptors were systematically screened for their contribution to ligand binding (37, $39,46)$. This method takes into account the potentially confounding effect of decreased expression of mutant receptors, and detects whether a given residue if beneficial, neutral or detrimental for the interaction. As expected, mutations of the DxL motif abolished binding. Other important residues were ligand-dependent. For example, binding to APRIL requires an aromatic residue in the hairpin (F78 of TACI; Y13 of BCMA), which is not required for BAFF binding (Figure 7). This residue is absent in BAFF-R that does not bind APRIL. Conversely, an arginine residue in the hairpin favors binding to BAFF, but is not required for binding to APRIL (R30 of BAFF-R; R84 of TACI). BCMA, which binds BAFF with low affinity, has a suboptimal residue in this position (H19). The one turn helix following the hairpin is also important in determining the specificity of the interactions. APRIL requires an arginine at the end of this helix (R27 of BCMA), whereas BAFF need a leucine (L38 of BAFF-R). Thus, L38 of BAFF-R favors BAFF binding but prevents APRIL binding. Conversely, R27 of BCMA favors APRIL binding and weakens BAFF binding. TACI adopts a different structure in this region, with P97 being the spatially corresponding residue that shows a preference for APRIL. In summary, BCMA, TACI and BAFF-R have an intrinsic tendency to bind BAFF and APRIL through the DxL motif, which is positively or negatively regulated in a ligand- 
dependent manner by various other residues. This explains the specificity of BAFF-R for BAFF and the preferential binding of BCMA to APRIL.

This structural knowledge was exploited to prepare an APRIL-specific BCMA by replacing a residue favorable for BAFF binding (I22) to one that enhances APRIL binding (I22K) (46) (Figure 7). In another application, anti-BAFF-R antibodies were selected by phage display on BAFF-R and submitted to affinity maturation in order to recognize both human and mouse BAFF-R with high affinity (47). One such antibody recognized the conserved DxL motif of BAFF-R, and shared several of its BAFF-Rbinding characteristics with BAFF. This antibody competed for BAFF binding and acted as a BAFF-R antagonist. Administration of this antibody to mice depleted various B cell populations by the dual mechanism of inhibiting BAFF signals and mediating antibodydependent cellular cytotoxicity (48). A similar but less marked effect was also observed in non-human primates (48).

\section{Receptor signaling}

\subsection{Signaling overview}

BAFF-R mediates much of the BAFF survival signals required to maintain the peripheral B cell population. BAFF can be functionally replaced for this purpose by the deletion of TRAF2 or TRAF3, or by the constitutive activation of either canonical or non-canonical $\mathrm{NF}-\kappa \mathrm{B}$ pathways (49-52). TRAF3 is the only known intracellular binding partner of BAFF-R (53). TRAF3 also negatively regulates the kinase NIK, which is apical to the non-canonical NF- $\mathrm{B}$ pathway (54). This suggests that BAFF-R engagement interferes with the negative function of TRAF3 on the NF-KB pathway, ultimately leading to the activation of this pathway and its pro-survival effects.

\subsection{TRAF binding}

A natural mutation affecting the last 8 amino acids of BAFF-R in A/WySnJ mice almost completely abolishes BAFF-R activity $(55,56)$. This mutation occurs close to a sequence conserved in both BAFF-R and BCMA that mediates binding to TRAF3 (53), strongly suggesting that TRAF3 regulates BAFF-R signaling. Indeed, mutation of the TRAF3binding sequence abolishes BAFF-R's ability to engage non-canonical NF-кB (57). 
TRAF-binding sequences in the intracellular portions of TNF receptors are linear peptides that usually bind either TRAF6, or TRAF1, 2, 3 and 5 . The strict specificity of BAFF-R for TRAF3 is therefore unusual, but the determinants for this specificity are now well understood $(57,58)$. The C-terminal portion of TRAF3 adopts a mushroom-like structure with the coiled-coil domain forming the stem and the homotrimeric TRAF-C domain forming the cap (Figure 9). The TRAF-binding sequence of BAFF-R wraps around the TRAF-C domain (58) (Figure 9). The overall similarity in size and symmetry of the extracellular BAFF - BAFF-R complex and of the intracellular BAFF-R - TRAF3 complex is striking. How BAFF-R exerts functional changes on TRAF-3 upon recruitment is not understood.

\section{Oligomerization-dependent signaling}

With the exception of delta-BAFF, whose transgenic over-expression demonstrated a dominant negative effect on B cell homeostasis (20), little is known regarding the physiological function of the various forms of BAFF and APRIL. The analysis of chimearic mice expressing BAFF either in bone marrow-derived cells or in non-bone marrow, radiation-resistant cells indicated that radiation-resistant cells can produce sufficient amounts of BAFF for maintaining the peripheral B cell population, whereas BAFF produced by bone marrow-derived cells may only provide a more local support to B cells (59). In any case, this study demonstrated the existence of different sources of BAFF with apparently different physiological effects. Whether various cell types produce different forms of BAFF, such a membrane-bound or soluble BAFF, remains to be studied.

A number of in vitro studies suggest that ligand oligomerization may modulate biological activity. In the original report, BAFF 60-mer had a modest two-fold co-stimulatory effect on human blood B cells that was not observed with a flap-deletion mutant (26). In mouse B cells, BAFF 60-mer was only moderately more active than BAFF 3-mer at costimulating B cell proliferation (36). The differential effects of BAFF 3-mer and 60-mer were analyzed in more detail using mouse cells (60). The co-stimulatory effect of BAFF did not rely on increased proliferation, but rather on increased B cell survival, consistent with its relatively minor effects. Survival was the result of a mixed signal originating 
from both BAFF-R and TACI. Whereas BAFF-R could be stimulated by both BAFF 3mer and BAFF 60-mer, TACI responded exclusively to BAFF 60-mer or other multimeric forms of BAFF. Thus, BAFF-mediated B cell co-stimulation is the result of two distinct signals: one originating from BAFF-R in response to any form of BAFF, and one originating from TACI in response to multimeric forms of BAFF (60). In plasmablasts, BAFF-mediated survival depended on TACI with little contribution of BAFF-R, and also required oligomeric forms of BAFF (60). In a similar way, TACI also responded to cross-linked APRIL, but not to APRIL 3-mer (27, 60). Importantly, TACI bound BAFF 3-mer and 60-mer equally well, but only signaled in response to the later. It is possible that binding of BAFF 3-mer to TACI is sufficient to recruit a single trimeric TRAF (see figure 9), which may not be able to signal as such. Indeed, cross-linking of TRAF2 and TRAF6 is required for efficient activation of the NF-KB pathway (61). Oligomeric forms of BAFF may allow signaling through TACI by enabling recruitment of several TRAFs in close proximity.

Interestingly, survival of human monocytes ex vivo was enhanced by BAFF in a TACIdependent manner (62). This effect was only observed with some commercial BAFF preparations but not others, although all of them supported B cell survival (62). These puzzling observations could be readily explained if the active preparations contained a proportion of oligomeric BAFF able to signal through TACI. This difference would not be detected on B cells that mainly respond through BAFF-R.

The finding that BAFF-R and TACI can respond to different forms of BAFF but transmit similar survival signals can help explain the paradoxical role of TACI in B cell biology. TACI-deficient mice indeed have impaired humoral responses despite their elevated B cell number $(12,13)$. Plasmablasts elicited in response to T-independent type-2 antigens appear to rely heavily on TACI for their survival (63), but cannot respond to BAFF 3-mer for lack of BAFF-R. Thus, deletion of TACI would penalize plasmablasts without preventing the majority of BAFF-R-expressing B cells to receive survival signals: these may even receive more, as TACI-deficient mice have elevated levels of circulating BAFF (60), leading to the observed enlargement of the B cell pool.

It is believed that APRIL is mainly released as a soluble cytokine (25). This form of APRIL can however not activate TACI, and is also probably poorly active on BCMA 
(60). Soluble APRIL released in vivo accumulates on proteoglycans (64). It is tempting to speculate that proteoglycan-bound APRIL is a biologically active form particularly important to support survival of antibody secreting cells in specialized environments such as the bone marrow or the intestinal mucosa $(65,66)$.

\section{Conclusions and open questions}

From a structural point of view, the BAFF - APRIL system is certainly one of the best characterized within the TNF family. Taken together, structural studies on BAFF, APRIL and their receptors have provided three highly unexpected surprises. The first one was the unique mode of receptor binding on a single ligand protomer, the second one was the discovery of the oligomeric form of BAFF 60-mer and the third one was the interaction of APRIL and TACI with glycosaminoglycans. These studies have not only provided a detailed molecular basis for the observed binding specificities between ligands and receptors, but also hinted at different mechanisms of action of trimeric and oligomeric BAFF and APRIL. The remaining open questions regarding structure are relatively few. They include the mapping of the proteoglycan binding site(s) of TACI, the mode of binding of full length TACI on BAFF 60-mer, the structural impact of exon 3 deletion in BAFF and the possibility of ligand-independent pre-association of TACI.

The analysis of receptor-deficient mice has taught us a lot on the role of these different receptors. However, little is known on the relative contributions of membrane-bound BAFF, soluble BAFF 3-mer and soluble BAFF 60-mer to the physiological functions of BAFF. With the recent discovery that these forms likely engage different sets of receptors, there is no doubt that the analysis of knock-in mice deficient for on or the other forms of BAFF and APRIL will yield interesting information on how these ligands control the various aspects of B cell biology they are implicated in.

\section{Acknowledgment}

This work was supported by the Swiss National Foundation for Scientific Research.

\section{Figure legends}

Figure 1: The complexity of the BAFF and APRIL system. 
Red arrowheads indicate furin consensus cleavage sites. Glycosaminoglycan side chains of proteoglycans are shown as thick wavy red lines. Two splicing isoforms of human TACI differing in the presence or absence of the first cystein-rich domain are depicted.

Figure 2: Sequence alignment of BAFF, BALM and APRIL.

The alignment shows sequences of the C-terminal portions of BAFF, BALM and APRIL of different species. $\beta$-strands as found in the crystal structure of human BAFF and mouse APRIL are schematized above and below the alignment, respectively. Hs: Homo sapiens (human). Mm: Mus musculus (mouse). Gg: Gallus gallus (chicken). Om: Oncorhynchus mykiss (rainbow trout). Ga: Gasterosteus aculeatus (threespine stickleback, a fish). The R-X-R/K-R furin consensus cleavage site is indicated (furin). Black dots: residues that are located within $4 \AA$ of another ligand protomer: These residues correspond roughly to residues involved in trimer formation. Gray squares: residues that are located within $4 \AA$ of a receptor (BAFF-R or BCMA for human BAFF; BCMA or TACI for mouse APRIL). These residues correspond roughly to those involved in receptor binding. Black diamonds: residues that are located within $4 \AA$ of another BAFF trimer. BAFF residues that are involved in trimer - trimer interactions for the formation of BAFF 60-mer are shaded gray. Cystein residues in $\beta$-sheets $E$ and $F$ involved in the formation of a disulfide bridge are shown in bold and linked by a bracket. The residue A120 of mouse APRIL that can be alternatively spliced is indicated by an arrow. The basic sequence of human and mouse APRIL involved in proteoglycan interaction is underlined (basic seq). "*” between the furin site and $\beta$-sheet A of mouse and trout BAFF indicate insertions of 29 and 20 amino acid residues, respectively.

Figure 3: Structural homology in the TNF family.

Ribbon representation of APRIL (mouse; pdb atomic coordinate file 1XU1), BAFF (human; 1OQE), CD40L (human; 1ALY), EDA1 (human; 1RJ7), lymphotoxin- $\alpha$ (human; LT $\alpha$; 1TNR), OX40L (mouse; 2HEW), RANKL (mouse; 1JTZ), TNF (human; 1TNF) and TRAIL (human; 1D4V). Structures are viewed along the three-fold symmetry axis, which is highlighted in the structure of TRAIL. The unusually long DE loop of BAFF is designated "flap". 
Figure 4: Structure of BAFF 60-mer.

Upper left pictures: Two individual BAFF trimers linked or not by the flap region. Upper middle picture: Space filling representation of BAFF 60-mer (1OTZ). Five trimers are colored in warm tones, and the remaining 15 are shown in pale blue. Upper right picture: Idem, but with 15 out of the 60 co-crystallized BAFF-R shown in green (1OTZ and 1POT). Bottom pictures: details of a trimer-trimer interaction, viewed from the center of the 60-mer (1OTZ). BAFF 1 and FLAP 1 belong to a given BAFF trimer, while BAFF 1', FLAP 1' and BAFF 2' belong to an adjacent BAFF trimer. Residues involved in three distinct groups of interactions are shown (salt bridges 1, salt brides 2 and hydrophobic core).

Figure 5: Model of APRIL bound to heparin and BCMA.

The structure of heparin (1FQ9) was manually positioned on that of the APRIL-BCMA complex (1XU2). Side chains of all basic residues in the upper portion of APRIL are shown in blue. In the side view (left picture), the BCMA-expressing cell would be at the bottom of the figure. The orange ball is a nickel atom probably originating from the purification process.

Figure 6: Sequence alignment of BCMA, TACI and BAFF-R.

$\beta$-sheets and helices found in the ligand-binding domain of human BAFF, human TACI CRD2 and human BAFF-R are shown above the sequences. Connectivity of disulfide bridges is indicated by brackets, and cystein residues are shown in bold. Both CRDs of TACI are shown individually. The DxL motif that is crucial for ligand binding is shaded.

Figure 7: Determinants of ligand-binding specificity in BAFF-R, TACI and BCMA. BAFF-R (1OQE; orange), TACI (1XU1; cyan) and BCMA (1OQD; purple) are shown twice. The upper part of the figure shows residues determining binding specificity to APRIL, and the lower part of the figure those residues important for binding to BAFF. These residues were identified by shotgun alanine scanning $(37,39,46)$. Side chains that 
are required for interactions are shown in green, whereas those that prevent or weaken interactions are shown in red. Disulfide bridges are colored in yellow.

Figure 8: Receptor - ligand binding interfaces in the TNF family.

Structures of 6 complexes of a ligand co-crystallized with its receptor are shown. The complexes are lymphotoxin- $\alpha /$ TNF-R1 (1TNR), TRAIL/DR5 (1DU3), OX40L/OX40 (2HEV), BAFF/BCMA (1OQD), BAFF/BAFF-R (1OQE) and APRIL/TACI (1XU1). Receptors are shown as yellow ribbons, whereas ligands are shown in the space filling representation, with one protomer in pale blue and another in pink. Ligand residues within $4 \AA$ of the receptor are shown in blue and red. They provide an estimation of residues involved in the interaction. In this representation, the transmembrane domain of the receptors would be at the bottom of the figure.

Figure 9: Comparison of BAFF - BAFF-R and BAFF-R - TRAF3 complexes.

Structure of a BAFF trimer (golden ribbon) bound to BAFF-R (green surface; 1OQE) and of the TRAF-binding sequence of the intracellular domain of BAFF-R (green surface) bound to the C-terminal portion of TRAF3 (purple ribbons, 2GKW). The cell membrane and the missing portion of BAFF-R are schematized as thick pink and green lines, respectively.

\section{References}

1. Gross JA, Dillon SR, Mudri S, et al. TACI-Ig neutralizes molecules critical for B cell development and autoimmune disease. impaired B cell maturation in mice lacking BLyS. Immunity 2001;15(2):289-302.

2. Mackay F, Woodcock SA, Lawton P, et al. Mice transgenic for BAFF develop lymphocytic disorders along with autoimmune manifestations. J Exp Med 1999;190(11):1697-710.

3. Schiemann B, Gommerman JL, Vora K, et al. An essential role for BAFF in the normal development of B cells through a BCMA-independent pathway. Science 2001;293(5537):2111-4.

4. Planelles L, Carvalho-Pinto CE, Hardenberg G, et al. APRIL promotes B-1 cell-associated neoplasm. Cancer Cell 2004;6(4):399-408.

5. Castigli E, Scott S, Dedeoglu F, et al. Impaired IgA class switching in APRIL-deficient mice. Proc Natl Acad Sci U S A 2004;101(11):3903-8.

6. Dillon SR, Gross JA, Ansell SM, Novak AJ. An APRIL to remember: novel TNF ligands as therapeutic targets. Nat Rev Drug Discov 2006;5(3):235-46.

7. Bossen C, Schneider P. BAFF, APRIL and their receptors: structure, function and signaling. Semin Immunol 2006;18(5):263-75.

8. Sasaki Y, Casola S, Kutok JL, Rajewsky K, Schmidt-Supprian M. TNF family member B cellactivating factor (BAFF) receptor-dependent and -independent roles for BAFF in B cell physiology. J Immunol 2004;173(4):2245-52. 
9. Shulga-Morskaya S, Dobles M, Walsh ME, et al. B cell-activating factor belonging to the TNF family acts through separate receptors to support B cell survival and T cell-independent antibody formation. $\mathrm{J}$ Immunol 2004;173(4):2331-41.

10. O'Connor BP, Raman VS, Erickson LD, et al. BCMA is essential for the survival of long-lived bone marrow plasma cells. J Exp Med 2004;199(1):91-8.

11. Seshasayee D, Valdez P, Yan M, Dixit VM, Tumas D, Grewal IS. Loss of TACI causes fatal lymphoproliferation and autoimmunity, establishing TACI as an inhibitory BLyS receptor. Immunity 2003;18(2):279-88.

12. von Bulow GU, van Deursen JM, Bram RJ. Regulation of the T-independent humoral response by TACI. Immunity 2001;14(5):573-82.

13. Yan M, Wang H, Chan B, et al. Activation and accumulation of B cells in TACI-deficient mice. Nat Immunol 2001;2(7):638-43.

14. Pan-Hammarstrom Q, Salzer U, Du L, et al. Reexamining the role of TACI coding variants in common variable immunodeficiency and selective IgA deficiency. Nat Genet 2007;39(4):429-30.

15. Salzer U, Chapel HM, Webster AD, et al. Mutations in TNFRSF13B encoding TACI are associated with common variable immunodeficiency in humans. Nat Genet 2005;37(8):820-8.

16. Castigli E, Wilson S, Garibyan L, et al. Reexamining the role of TACI coding variants in common variable immunodeficiency and selective IgA deficiency. Nat Genet 2007;39(4):430-1.

17. Castigli E, Wilson SA, Garibyan L, et al. TACI is mutant in common variable immunodeficiency and IgA deficiency. Nat Genet 2005;37(8):829-34.

18. Kothlow S, Morgenroth I, Graef Y, et al. Unique and conserved functions of B cell-activating factor of the TNF family (BAFF) in the chicken. Int Immunol 2007;19(2):203-15.

19. Glenney GW, Wiens GD. Early diversification of the TNF superfamily in teleosts: genomic characterization and expression analysis. J Immunol 2007;178(12):7955-73.

20. Gavin AL, Duong B, Skog P, et al. deltaBAFF, a splice isoform of BAFF, opposes full-length BAFF activity in vivo in transgenic mouse models. J Immunol 2005;175(1):319-28.

21. Pradet-Balade B, Medema JP, Lopez-Fraga M, et al. An endogenous hybrid mRNA encodes TWEPRIL, a functional cell surface TWEAK-APRIL fusion protein. Embo J 2002;21(21):5711-20.

22. Bossen C, Ingold $\mathrm{K}$, Tardivel A, et al. Interactions of tumor necrosis factor (TNF) and TNF receptor family members in the mouse and human. J Biol Chem 2006;281(20):13964-71.

23. Moore PA, Belvedere O, Orr A, et al. BLyS: member of the tumor necrosis factor family and B lymphocyte stimulator. Science 1999;285(5425):260-3.

24. Schneider P, MacKay F, Steiner V, et al. BAFF, a novel ligand of the tumor necrosis factor family, stimulates B cell growth. J Exp Med 1999;189(11):1747-56.

25. Lopez-Fraga M, Fernandez R, Albar JP, Hahne M. Biologically active APRIL is secreted following intracellular processing in the Golgi apparatus by furin convertase. EMBO Rep 2001;2(10):945-51.

26. Liu Y, Xu L, Opalka N, Kappler J, Shu HB, Zhang G. Crystal structure of sTALL-1 reveals a viruslike assembly of TNF family ligands. Cell 2002;108(3):383-94.

27. Ingold K, Zumsteg A, Tardivel A, et al. Identification of proteoglycans as the APRIL-specific binding partners. J Exp Med 2005;201(9):1375-83.

28. Hendriks J, Planelles L, de Jong-Odding J, et al. Heparan sulfate proteoglycan binding promotes APRIL-induced tumor cell proliferation. Cell Death Differ 2005;12(6):637-48.

29. Roschke V, Sosnovtseva S, Ward CD, et al. BLyS and APRIL form biologically active heterotrimers that are expressed in patients with systemic immune-based rheumatic diseases. J Immunol 2002;169(8):4314-21.

30. Bodmer JL, Schneider P, Tschopp J. The molecular architecture of the TNF superfamily. Trends Biochem Sci 2002;27(1):19-26.

31. Wallweber HJ, Compaan DM, Starovasnik MA, Hymowitz SG. The crystal structure of a proliferationinducing ligand, APRIL. J Mol Biol 2004;343(2):283-90.

32. Oren DA, Li Y, Volovik Y, et al. Structural basis of BLyS receptor recognition. Nat Struct Biol 2002;9(4):288-92.

33. Karpusas M, Cachero TG, Qian F, et al. Crystal structure of extracellular human BAFF, a TNF family member that stimulates B lymphocytes. J Mol Biol 2002;315(5):1145-54.

34. Liu Y, Hong X, Kappler J, et al. Ligand-receptor binding revealed by the TNF family member TALL1. Nature 2003;423(6935):49-56. 
35. Kim HM, Yu KS, Lee ME, et al. Crystal structure of the BAFF-BAFF-R complex and its implications for receptor activation. Nat Struct Biol 2003;10(5):342-8.

36. Cachero TG, Schwartz IM, Qian F, et al. Formation of virus-like clusters is an intrinsic property of the tumor necrosis factor family member BAFF (B cell activating factor). Biochemistry 2006;45(7):200613.

37. Gordon NC, Pan B, Hymowitz SG, et al. BAFF/BLyS receptor 3 comprises a minimal TNF receptorlike module that encodes a highly focused ligand-binding site. Biochemistry 2003;42(20):5977-83.

38. Couchman JR. Syndecans: proteoglycan regulators of cell-surface microdomains? Nat Rev Mol Cell Biol 2003;4(12):926-37.

39. Hymowitz SG, Patel DR, Wallweber HJ, et al. Structures of APRIL-receptor complexes: like BCMA, TACI employs only a single cysteine-rich domain for high affinity ligand binding. J Biol Chem 2005;280(8):7218-27.

40. Bischof D, Elsawa SF, Mantchev G, et al. Selective activation of TACI by syndecan-2. Blood 2006;107(8):3235-42.

41. Banner DW, D'Arcy A, Janes W, et al. Crystal structure of the soluble human $55 \mathrm{kd}$ TNF receptorhuman TNF beta complex: implications for TNF receptor activation. Cell 1993;73(3):431-45.

42. Mongkolsapaya J, Grimes JM, Chen N, et al. Structure of the TRAIL-DR5 complex reveals mechanisms conferring specificity in apoptotic initiation. Nat Struct Biol 1999;6(11):1048-53.

43. Hymowitz SG, Christinger HW, Fuh G, et al. Triggering cell death: the crystal structure of Apo2L/TRAIL in a complex with death receptor 5. Mol Cell 1999;4(4):563-71.

44. Compaan DM, Hymowitz SG. The crystal structure of the costimulatory OX40-OX40L complex. Structure 2006;14(8):1321-30.

45. Kayagaki N, Yan M, Seshasayee D, et al. BAFF/BLyS receptor 3 binds the B cell survival factor BAFF ligand through a discrete surface loop and promotes processing of NF-kappaB2. Immunity 2002;17(4):515-24.

46. Patel DR, Wallweber HJ, Yin J, et al. Engineering an APRIL-specific B cell maturation antigen. J Biol Chem 2004;279(16):16727-35.

47. Lee CV, Hymowitz SG, Wallweber HJ, et al. Synthetic anti-BR3 antibodies that mimic BAFF binding and target both human and murine B cells. Blood 2006;108(9):3103-11.

48. Lin WY, Gong Q, Seshasayee D, et al. Anti-BR3 antibodies: a new class of B-cell immunotherapy combining cellular depletion and survival blockade. Blood 2007;110(12):3959-67.

49. Grech AP, Amesbury M, Chan T, Gardam S, Basten A, Brink R. TRAF2 differentially regulates the canonical and noncanonical pathways of NF-kappaB activation in mature B cells. Immunity 2004;21(5):629-42.

50. Xie P, Stunz LL, Larison KD, Yang B, Bishop GA. Tumor necrosis factor receptor-associated factor 3 is a critical regulator of B cell homeostasis in secondary lymphoid organs. Immunity 2007;27(2):25367.

51. Sasaki Y, Derudder E, Hobeika E, et al. Canonical NF-kappaB activity, dispensable for B cell development, replaces BAFF-receptor signals and promotes B cell proliferation upon activation. Immunity 2006;24(6):729-39.

52. Enzler T, Bonizzi G, Silverman GJ, et al. Alternative and classical NF-kappa B signaling retain autoreactive B cells in the splenic marginal zone and result in lupus-like disease. Immunity 2006;25(3):403-15.

53. Xu LG, Shu HB. TNFR-associated factor-3 is associated with BAFF-R and negatively regulates BAFF-R-mediated NF-kappa B activation and IL-10 production. J Immunol 2002;169(12):6883-9.

54. Liao G, Zhang M, Harhaj EW, Sun SC. Regulation of the NF-kappaB-inducing kinase by tumor necrosis factor receptor-associated factor 3-induced degradation. J Biol Chem 2004;279(25):26243-50.

55. Thompson JS, Bixler SA, Qian F, et al. BAFF-R, a newly identified TNF receptor that specifically interacts with BAFF. Science 2001;293(5537):2108-11.

56. Yan M, Brady JR, Chan B, et al. Identification of a novel receptor for B lymphocyte stimulator that is mutated in a mouse strain with severe B cell deficiency. Curr Biol 2001;11(19):1547-52.

57. Morrison MD, Reiley W, Zhang M, Sun SC. An atypical tumor necrosis factor (TNF) receptorassociated factor-binding motif of B cell-activating factor belonging to the TNF family (BAFF) receptor mediates induction of the noncanonical NF-kappaB signaling pathway. J Biol Chem 2005;280(11):10018-24. 
58. Ni CZ, Oganesyan G, Welsh K, et al. Key molecular contacts promote recognition of the BAFF receptor by TNF receptor-associated factor 3: implications for intracellular signaling regulation. $\mathrm{J}$ Immunol 2004;173(12):7394-400.

59. Gorelik L, Gilbride K, Dobles M, Kalled SL, Zandman D, Scott ML. Normal B cell homeostasis requires B cell activation factor production by radiation-resistant cells. J Exp Med 2003;198(6):937-45.

60. Bossen C, Cachero TG, Tardivel A, et al. TACI, unlike BAFF-R, is solely activated by oligomeric BAFF and APRIL to support survival of activated B cells and plasmablasts. Blood 2008;111(3):100412.

61. Baud V, Liu ZG, Bennett B, Suzuki N, Xia Y, Karin M. Signaling by proinflammatory cytokines: oligomerization of TRAF2 and TRAF6 is sufficient for JNK and IKK activation and target gene induction via an amino-terminal effector domain. Genes Dev 1999;13(10):1297-308.

62. Chang SK, Arendt BK, Darce JR, Wu X, Jelinek DF. A role for BLyS in the activation of innate immune cells. Blood 2006;108(8):2687-94.

63. Mantchev GT, Cortesao CS, Rebrovich M, Cascalho M, Bram RJ. TACI is required for efficient plasma cell differentiation in response to T-independent type 2 antigens. J Immunol 2007;179(4):22828.

64. Schwaller J, Schneider P, Mhawech-Fauceglia P, et al. Neutrophil-derived APRIL concentrated in tumor lesions by proteoglycans correlates with human B-cell lymphoma aggressiveness. Blood 2007;109(1):331-8.

65. Belnoue E, Pihlgren M, McGaha TL, et al. APRIL is critical for plasmablast survival in the bone marrow and poorly expressed by early life bone marrow stromal cells. Blood 2008.

66. $\mathrm{Xu} \mathrm{W}, \mathrm{He} \mathrm{B}$, Chiu A, et al. Epithelial cells trigger frontline immunoglobulin class switching through a pathway regulated by the inhibitor SLPI. Nat Immunol 2007;8(3):294-303. 


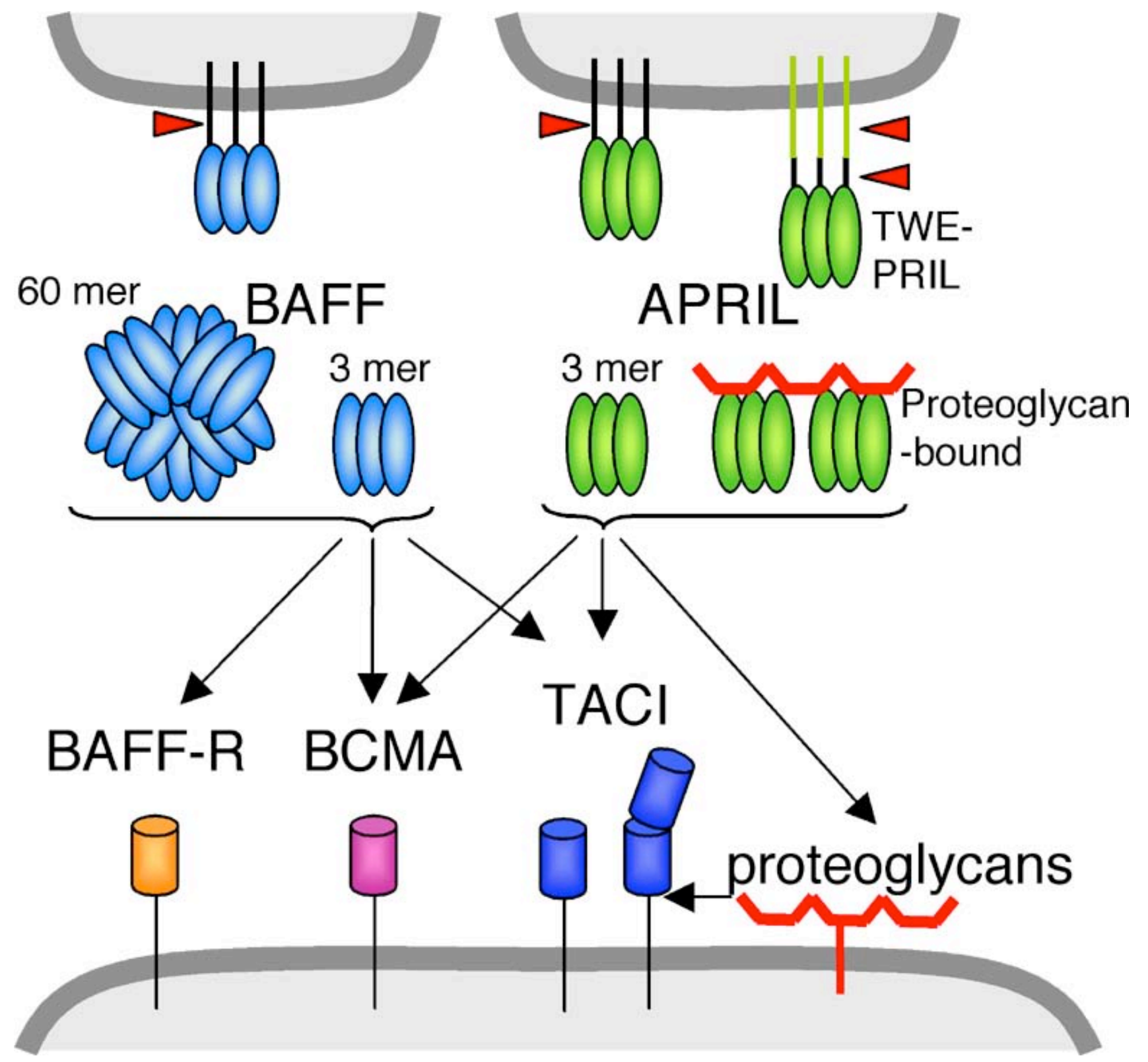

Schneider Fig1 


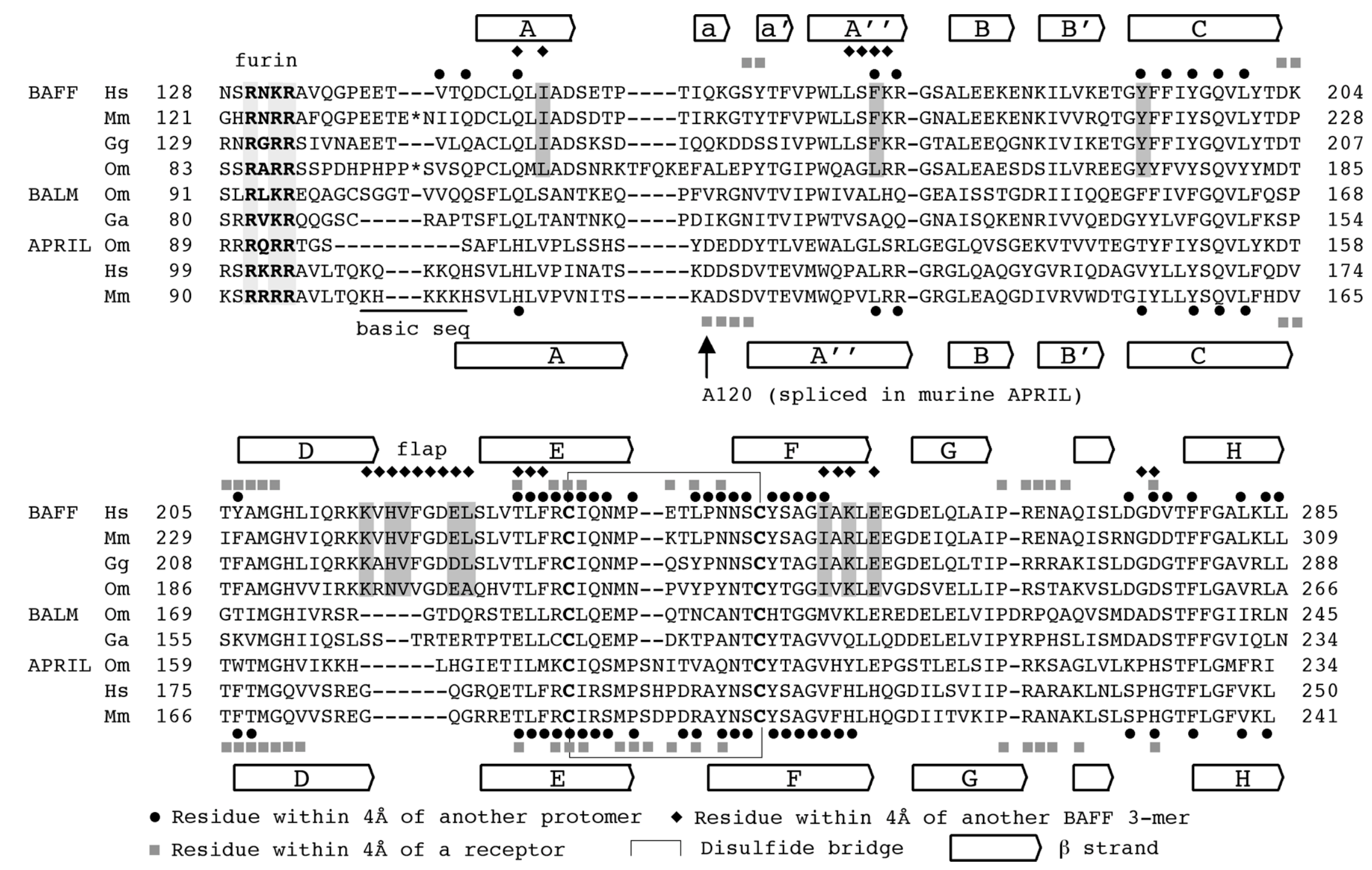

Schneider Fig2 

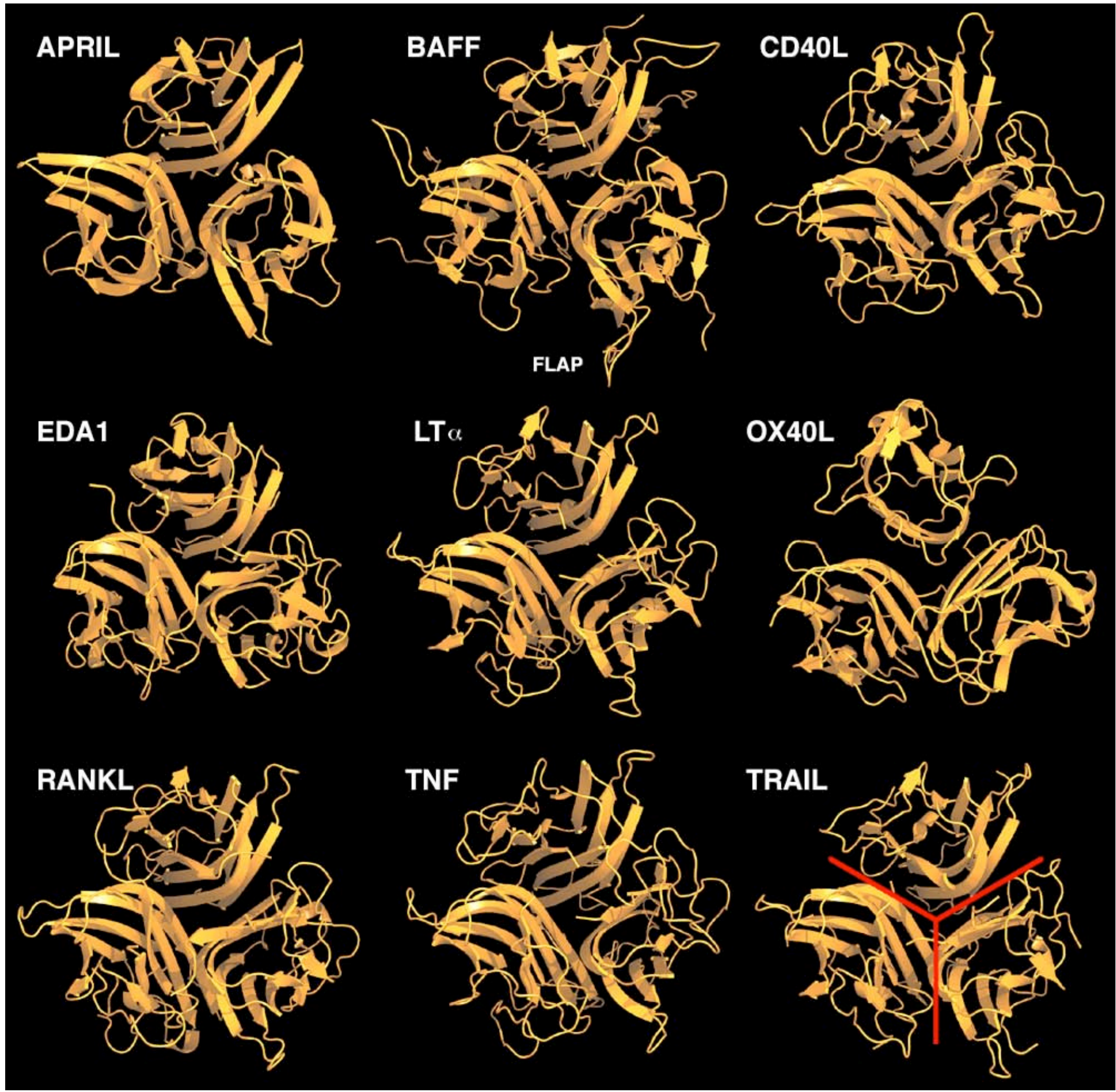

Schneider Fig3 


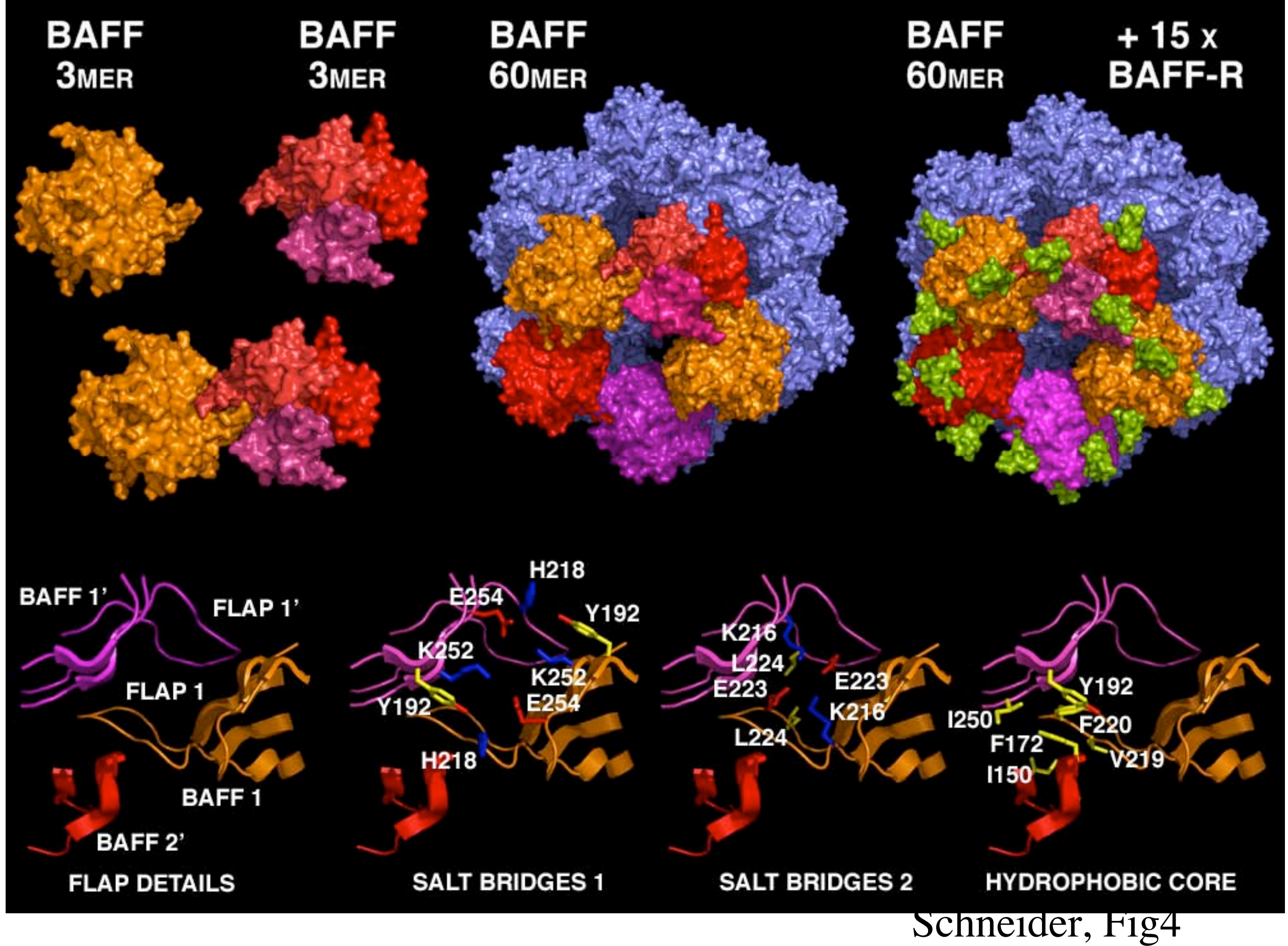






TOP VIEW



Schneider, Fig5 
hBCMA

mBCMA

hTACI Crd2 69

mTACI Crd2 41

hTACI Crd1 27

mTACI crd1

$\overbrace{\text { DxL motif }}^{\beta \text {-hairpin }} \overbrace{\text { helix-loop-helix }}^{\text {helo }}$

1 MLQMAGQCSQ--NEYFDSLLHACIPCQLRCSSNTPPLTCQRYCNASVTNSVKGT

$\beta 2$ h1

1 MAQQCFH--SEYFDSLLHACKPCHLRCS-N-PPATCQPYCDPSVTSSVKGT

hBAFFR

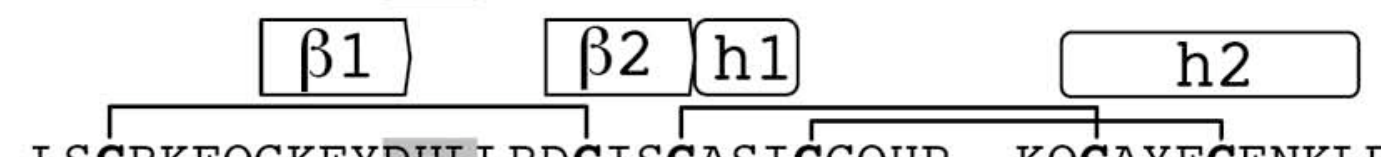

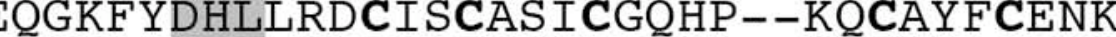
TGVAMRSCPE--EQYWDPLLGTCMSCKTICNHQS-QRTCAAFCRS

1 MAMAFCPK--DQYWDSSRKSCVSCALTCSQRS-QRTCTDFCKF

$\beta 1 \quad \beta 2$ h1

MBAFFR

12 DAPAPTPCVP--AECFDLLVRHCVACGLLRTPRPKPAGASSPAPRTALQPQESV

\section{Schneider Fig6}


RESIDUES BINDING APRIL (GREEN=FAVORABLE, RED=UNFAVORABLE) BAFF-R TACI BCMA
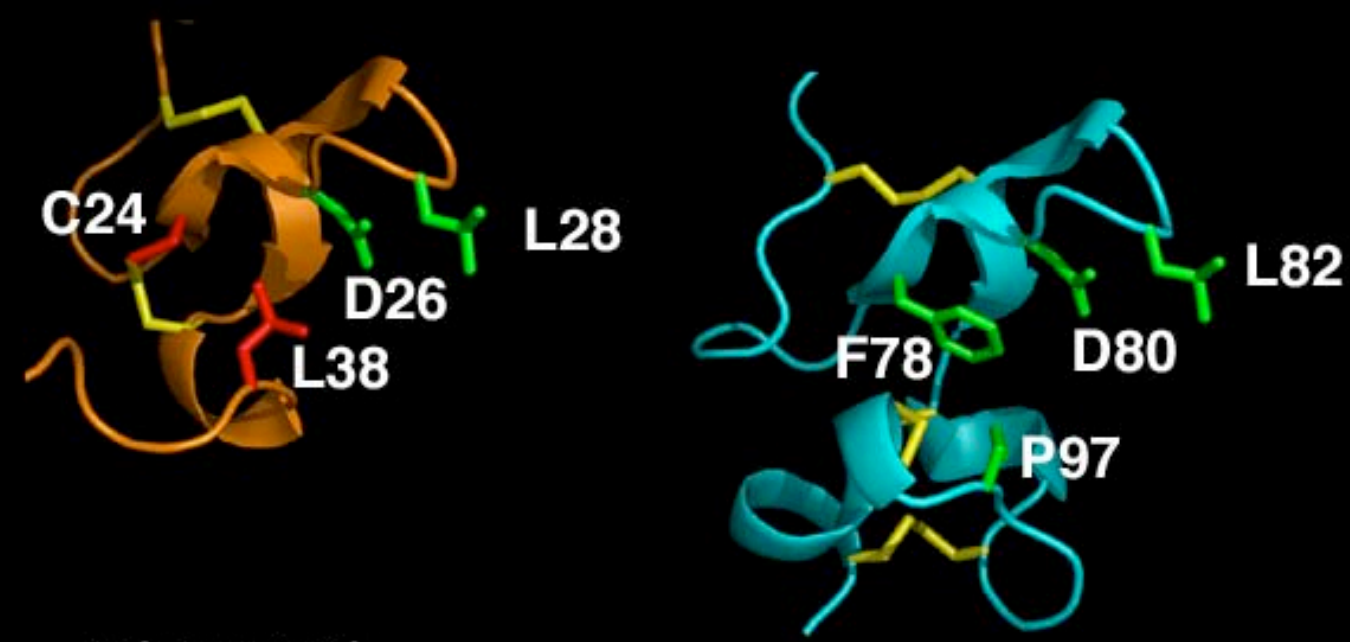

NO BINDING

HIGH AFFINITY BINDING



HIGH AFFINITY BINDING

RESIDUES BINDING BAFF (GREEN=FAVORABLE, RED=UNFAVORABLE) BAFF-R TACI
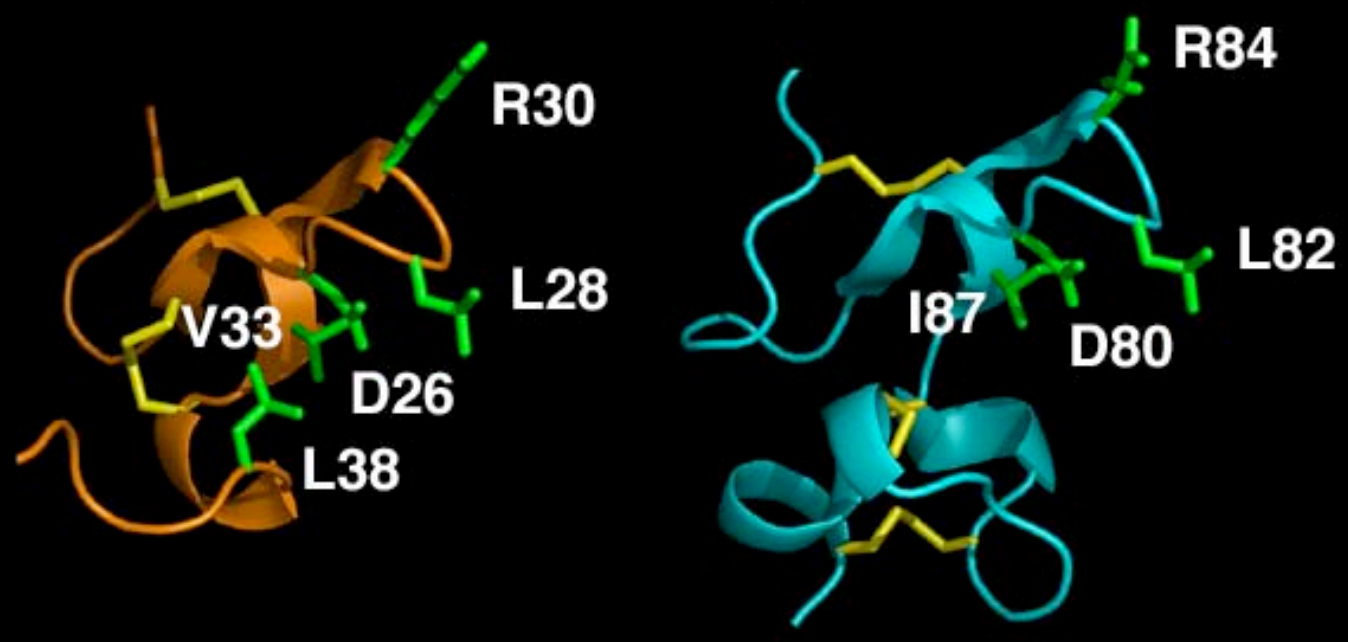
BCMA

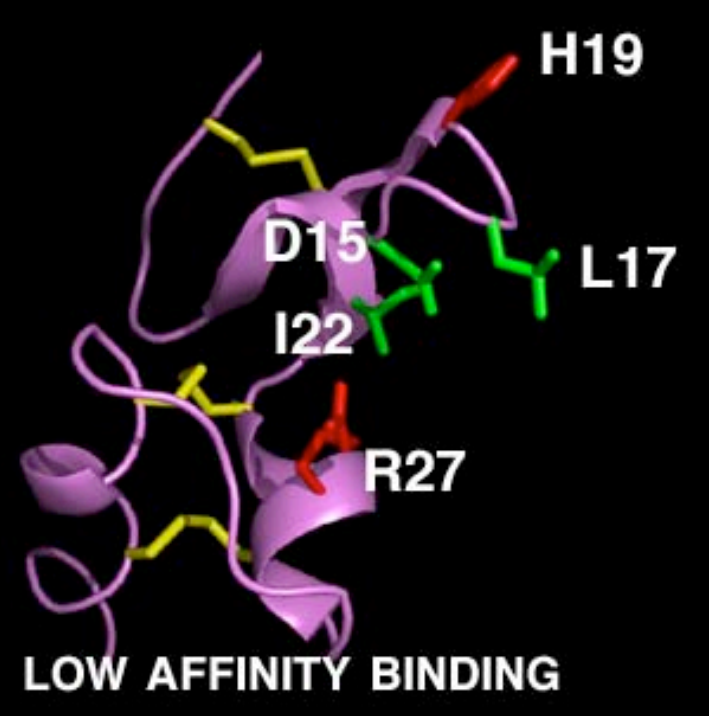

Schneider Fig7 


\section{LT $\alpha$ / TNFR1}

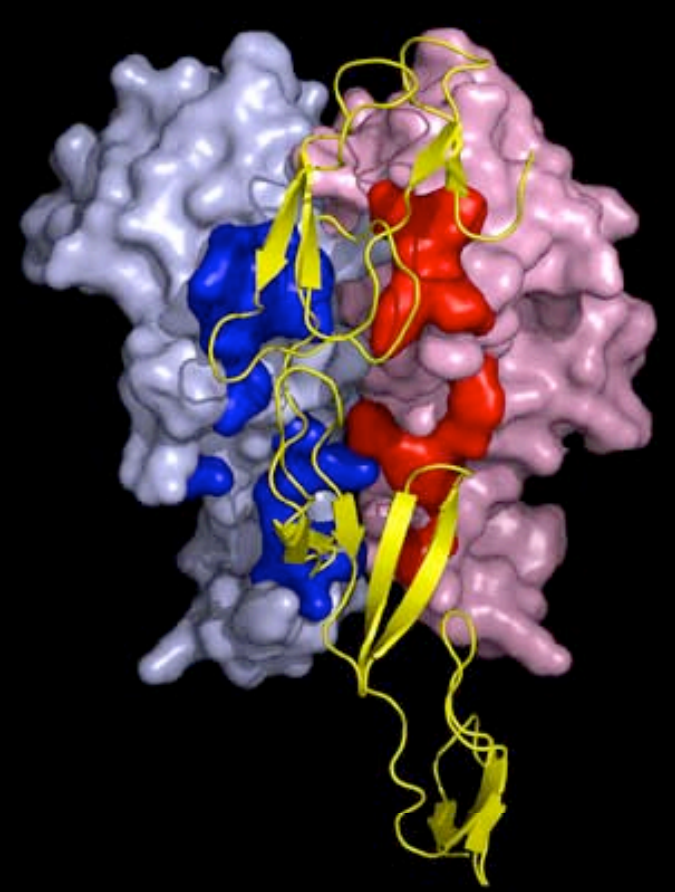

BAFF / BCMA

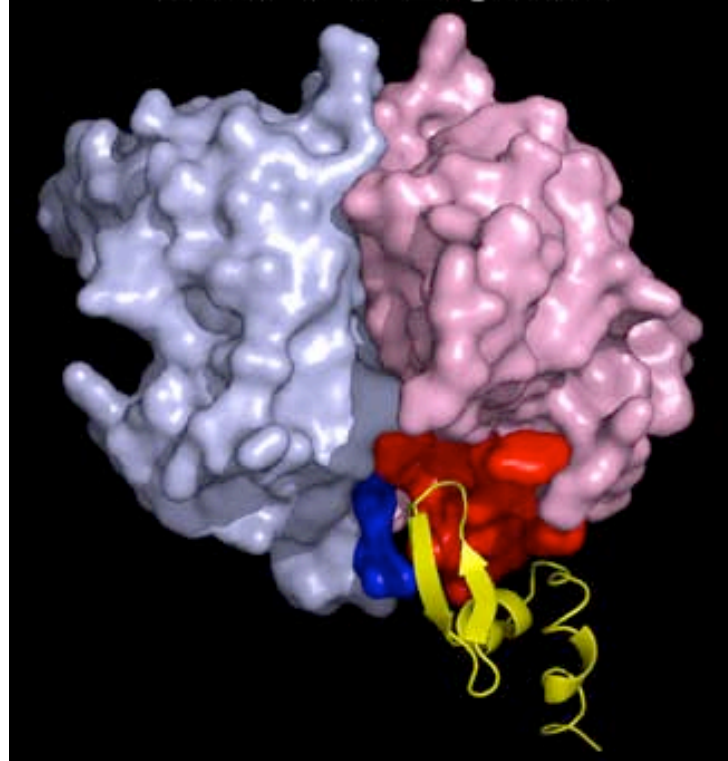

TRAIL / DR5

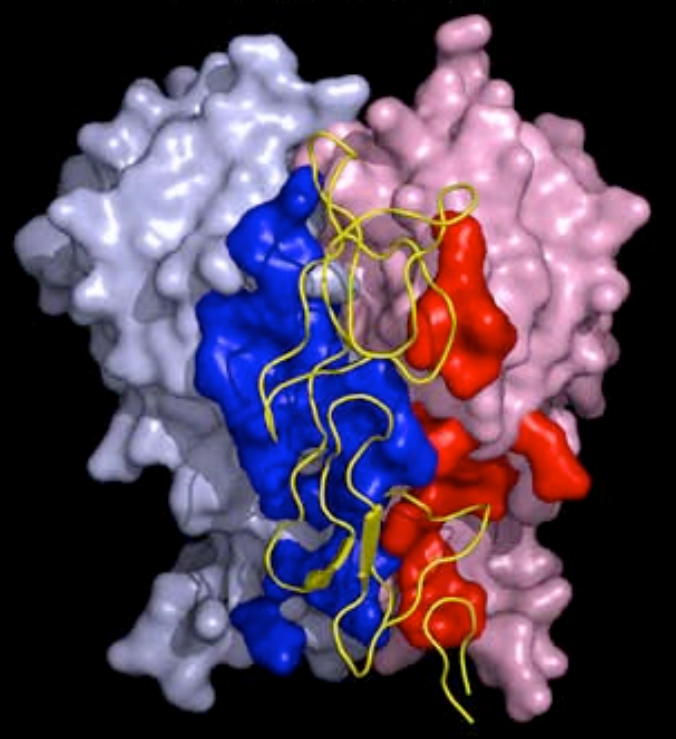

BAFF / BAFF-R

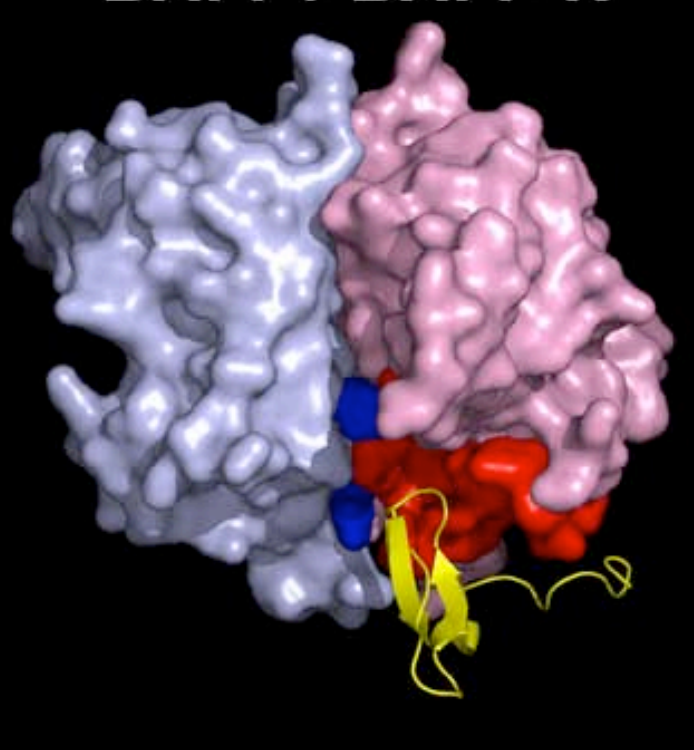

OX40L / OX40

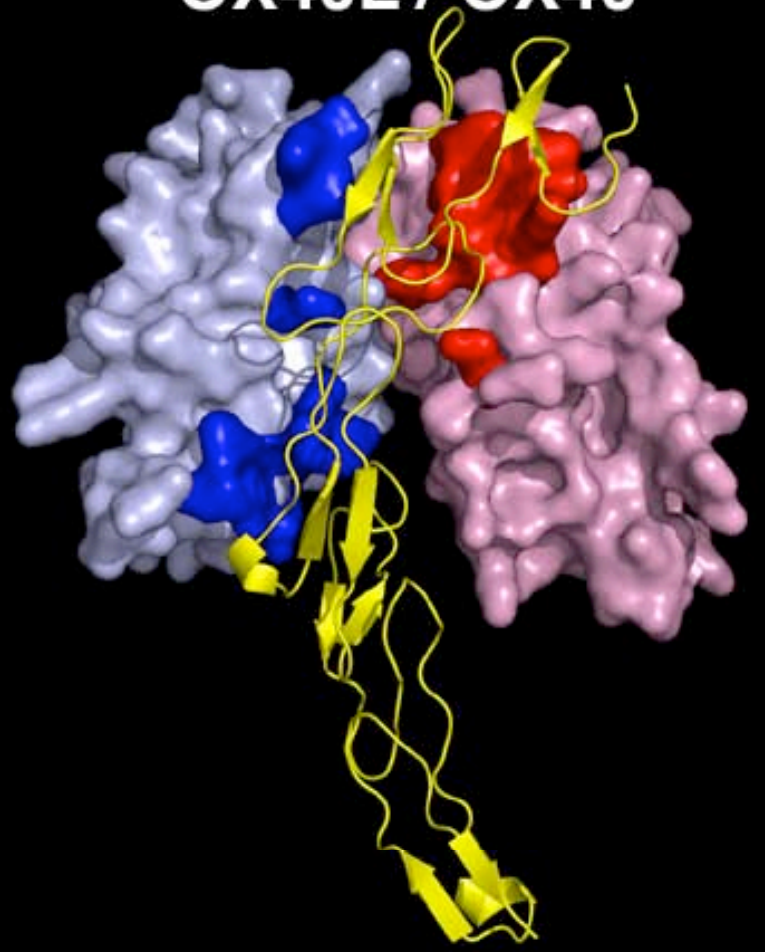

APRIL / TACI

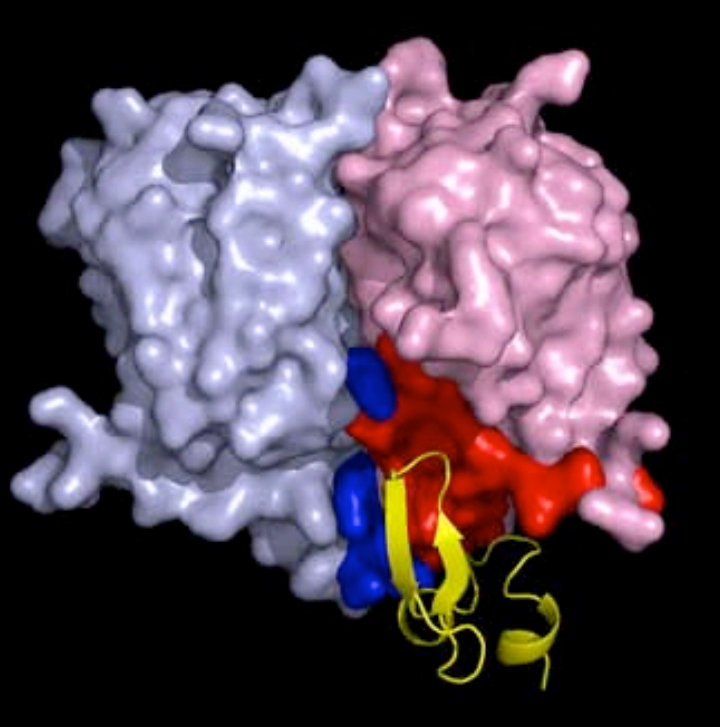

Schneider Fig8 




\section{TOP VIEW}
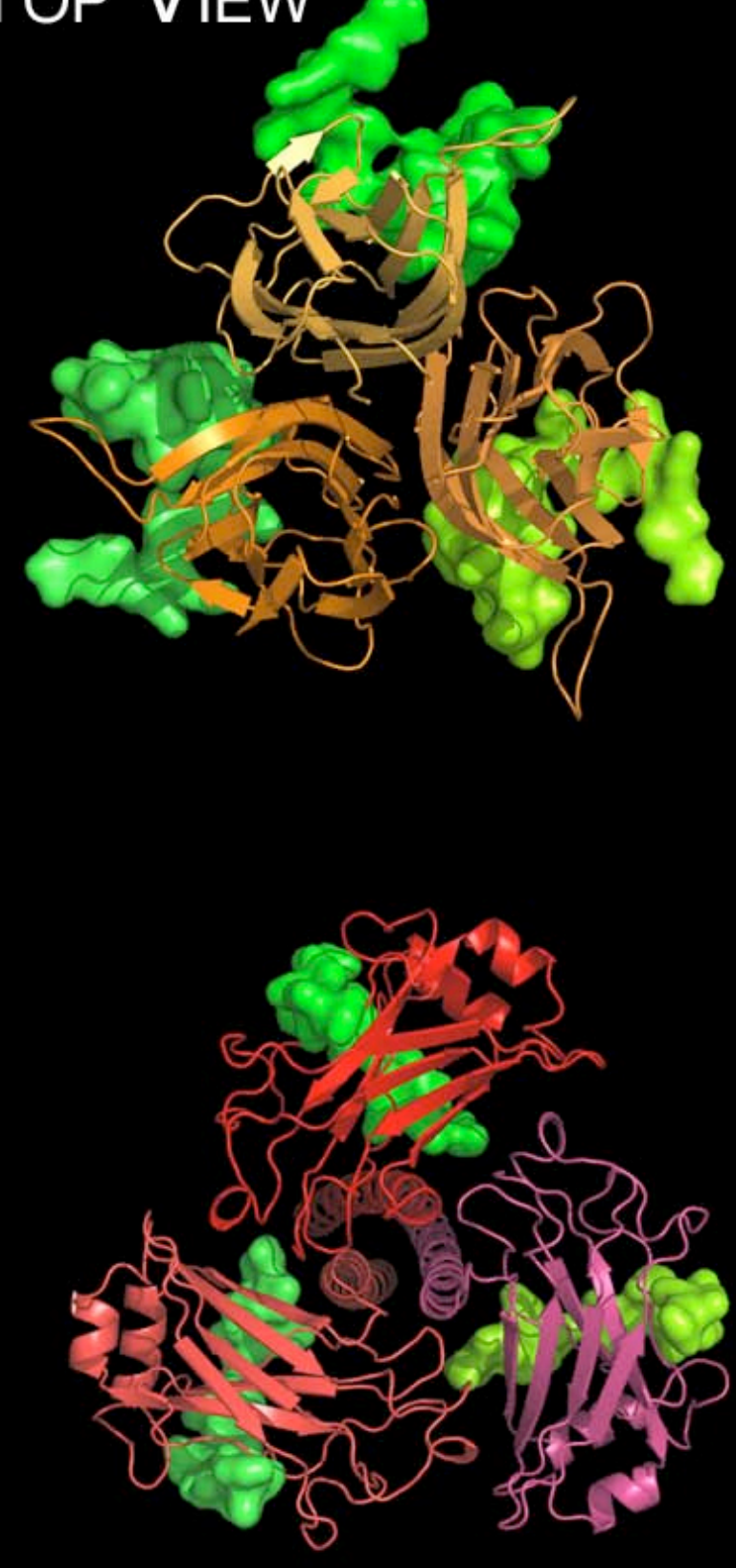

Schneider, Fig9 